Revista Española de Nutrición Humana y Dietética Spanish Journal of Human Nutrition and Dietetics

\title{
INVESTIGACIÓN
}

\section{Cambios en las prácticas culinarias y gastronómicas durante el confinamiento de la COVID-19 en la provincia de Alicante}

\section{Culinary and Gastronomic Practices During the COVID-19 confinement in the Alicante province}

\footnotetext{
María Tormo-Santamaría ${ }^{a, b}$, Luis Català-Oltra ${ }^{c, d, *}$, Alexandre Peretó-Rovira ${ }^{e}$, Ángeles RuízGarcía $^{\mathrm{a}, \mathrm{e}}$ and Josep Bernabeu-Mestre ${ }^{\mathrm{a}, \mathrm{b}, \mathrm{e}}$.

a Cátedra Carmencita de Estudios del Sabor Gastronómico, Departament d'Infermeria Comunitària, Medicina Preventiva i Salut Pública i Història de la Ciència, Universitat d'Alacant, San Vicente del Raspeig, España.

b Grup Balmis d'investigació en Salut Comunitària i Història de la Ciència, Universitat d'Alacant, San Vicente del Raspeig, España.

' Departament de Sociologia II, Universitat d'Alacant, San Vicente del Raspeig, España.

d CRITERI: Socioeconomia Crítica i Territori, Universitat d'Alacant, San Vicente del Raspeig, España.

e Gasterra, Centre de Gastronomía del Mediterrani, Universitat d'Alacant, Dénia, España.

* luis.catala@ua.es
}

Editor asignado: Samuel Durán Agüero. Universidad San Sebastián, Chile.

Recibido: 03/08/2020; Aceptado: 27/11/2020; Publicado: 28/12/2020

CITA: Tormo-Santamaría M, Català-Oltra L, Peretó-Rovira A, Ruíz-García A, Bernabeu-Mestre J. Cambios en las prácticas culinarias y gastronómicas durante el confinamiento de la COVID-19 en la provincia de Alicante. Rev Esp Nutr Hum Diet. 2021; 25 (Supl. 2): e1130. doi: 10.14306/renhyd.25.S2.1130

La Revista Española de Nutrición Humana y Dietética se esfuerza por mantener a un sistema de publicación continua, de modo que los artículos se publican antes de su formato final (antes de que el número al que pertenecen se haya cerrado y/o publicado). De este modo, intentamos poner los artículos a disposición de los lectores/usuarios lo antes posible.

The Spanish Journal of Human Nutrition and Dietetics strives to maintain a continuous publication system, so that the articles are published before its final format (before the number to which they belong is closed and/or published). In this way, we try to put the articles available to readers/users as soon as possible. 


\section{RESUMEN}

Introducción: El confinamiento generado por el estado de alarma decretado a causa de la COVID19 obligó a la ciudadanía a permanecer en sus casas salvo causa justificada y limitó el acceso a los servicios de restauración colectiva. Estas restricciones supusieron el cambio de hábitos y rutinas de la población, lo que se tradujo en más actividad culinaria en los domicilios. El objetivo del presente estudio es conocer el impacto del confinamiento sobre el comportamiento alimentario estudiando los cambios en las prácticas culinarias y gastronómicas experimentadas por la población alicantina.

Material y métodos: Estudio observacional transversal aunque con enfoque diacrónico a partir de la percepción y experiencias de los entrevistados: antes/durante el confinamiento, y muestreo no probabilístico de conveniencia de la población de 18 a 69 años reclutada de forma electrónica entre el 28 de abril y el 10 de mayo de 2020, semanas 7-8 de confinamiento, en la provincia de Alicante ( $n=679)$. Análisis estadístico univariable, bivariable y multivariable de los datos mediante el paquete estadístico SPSS. Uso de pruebas no paramétricas al asumir la condición de muestras emparejadas para las respuestas antes (muestra teórica 1) y durante el confinamiento (muestra teórica 2).

Resultados: El 70\% cocinó con más frecuencia y dedicó más tiempo a hacerlo. Más del 35\% amplió sus conocimientos culinarios con un incremento de consulta a la web y redes sociales. Durante el confinamiento predominó la cocina tradicional valenciana y los postres fueron las elaboraciones preferidas. El cocinado en grupo aumentó en más de 11 puntos porcentuales, al igual que comer en compañía, aunque persiste el uso de dispositivos audiovisuales durante el acto de comer. El $84 \%$ modificó su percepción sobre algún aspecto relacionado con la alimentación. Las diferencias entre el comportamiento previo a la pandemia y el que se desarrolló durante el confinamiento se han revelado significativas estadísticamente en la mayoría de las variables testadas.

Conclusiones: Durante el confinamiento, el aumento de la actividad culinaria se tradujo en una apuesta por la cocina tradicional en detrimento de los platos precocinados, más convivialidad en el acto de comer y un mayor compromiso con el comercio y el consumo de alimentos de proximidad y la sustentabilidad del sistema alimentario.

Palabras clave: Culinaria; Conducta Alimentaria; Infecciones por Coronavirus; Confinamiento; Gastronomía; COVID-19; síndrome respiratorio agudo severo coronavirus 2; SARS-CoV-2. 


\section{ABSTRACT}

Introduction: The confinement generated by the state of alarm decreed by the COVID-19, forced citizens to stay in their homes except for justified cause and limited access to collective catering services. These restrictions led to a change in the habits and routines of the population, which translated into more culinary activity at home. The objective of this study is to know the impact of confinement on eating behavior, studying the changes in culinary and gastronomic practices experienced by the Alicante population.

Material and methods: Observational cross-sectional, although with a diachronic approach based on the perception and experiences of the interviewees: before/during confinement, and nonprobabilistic convenience sampling of the population aged 18-69 years recruited electronically between 28 April and 10 May 2020 (weeks 7-8 of confinement) in the province of Alicante ( $n=$ 679). Univariate, bivariate, and multivariate statistical analysis of the data using the SPSS statistical package. Use of nonparametric tests by assuming the condition of paired samples for the responses before (sample 1) and during confinement (sample 2).

Results: 70\% cooked more often and spent more time cooking. More than 35\% expanded their culinary knowledge, with an increase in web browsing and social networking. During the confinement, traditional Valencian cuisine predominated and desserts were the preferred preparations. Group cooking increased by more than 11 percentage points, as did eating in company, although the use of audio-visual devices during the act of eating persisted. $84 \%$ changed their perception about some aspect related to food. The differences between the behavior prior to the pandemic and the one that developed during confinement have been shown to be statistically significant in most of the variables tested.

Conclusions: During the confinement, the increase in culinary activity translated into a commitment to traditional cuisine to the detriment of pre-cooked dishes, more conviviality in the act of eating, and a greater commitment to trade and consumption of local foods and the sustainability of the food system.

Keywords: Cooking; Feeding Behavior; Coronavirus Infections; Confinement; Gastronomy; COVID-19; severe acute respiratory syndrome coronavirus 2; SARS-CoV-2. 


\section{INTRODUCCIÓN}

La Organización Mundial de la Salud (OMS) acordó decretar la emergencia internacional por el brote de coronavirus detectado en la ciudad china de Wuhan el 30 de enero de 2020. El 11 de marzo ante los alarmantes niveles de propagación de la enfermedad y por su gravedad, la OMS determinaba que la COVID-19 podía caracterizarse como una pandemia ${ }^{1}$.

En consonancia con la evolución del contexto internacional y de los niveles de contagio alcanzados en España, el estado de alarma, a consecuencia de la pandemia de coronavirus fue declarado el 14 de marzo de $2020^{2}$ con vigencias temporales diferentes en cada comunidad autónoma en función de la evolución epidemiológica. En el caso de la Comunidad Valenciana se prolongó hasta el 10 de mayo la conocida como fase 0 del confinamiento. Cabe destacar que respecto a las medidas adoptadas por otros países del ámbito europeo, España fue uno de los más restrictivos. A pesar de ello, en esa fase 0 las muertes se dispararon un $74 \%$ respecto a las contabilizadas el año anterior en las mismas fechas y en la actualidad ocupa el tercer puesto de la Unión Europea en número de fallecidos por coronavirus por cada 100.000 habitantes ${ }^{3,4}$.

Como ha ocurrido en muchos otros ámbitos, los hábitos alimentarios también se han visto afectados por la aparición de la COVID-195. A consecuencia de las medidas de confinamiento, se ha visto alterado entre otros elementos, uno de los fenómenos más característicos de la modernización alimentaria de las últimas décadas: el auge que habría alcanzado comer fuera del hogar ya fuese por razones de trabajo o de ocio.

En el caso español, como apuntan Díaz-Méndez y col. ${ }^{6}$ con datos de una encuesta estatal de 2014, comer fuera de casa reúne unas características propias. En España este comportamiento lleva aparejado un alto grado de sociabilidad y permite romper con la rutina diaria que afecta al hogar y al trabajo.

En términos de acceso a los productos alimenticios no llegó a producirse desabastecimiento salvo por conductas de acaparamiento de algunos productos, como ocurrió con la levadura o la harina en las grandes superficies ${ }^{7,8}$.

En el mismo Real Decreto de 14 de marzo por el que se declara el estado de alarma para la gestión de la situación de crisis sanitaria ocasionada por el COVID-19, se establecían, en concreto en el artículo 15 todo un conjunto de medidas encaminadas a garantizar el abastecimiento alimentario en los lugares de consumo y el funcionamiento de los servicios de centros de producción, así como la distribución. Durante la fase cero del confinamiento estaba permitido junto a otras actividades de carácter esencial, circular por las vías de uso público para la adquisición de alimentos, además de productos farmacéuticos y de primera necesidad. 
Durante el estado de alarma no estuvo permitida la actividad hostelera y de restauración, aunque sí se contemplaba exclusivamente el servicio de entrega a domicilio ${ }^{2}$. Esta circunstancia redujo notablemente el consumo de alimentos elaborados en establecimientos de restauración fuera del hogar y propició que se incrementasen las actividades culinarias en los domicilios durante los primeros cincuenta y ocho días del confinamiento que siguió al estado de alarma. Los cambios inesperados que la pandemia provocó en los hábitos de consumo afectaron a la forma de cocinar y el tipo de cocina, las pautas de consumo, las prácticas gastronómicas o la sociabilidad ligada a la práctica de comer fuera de casa, entre otras consecuencias. La intuición de que se estaba produciendo este tipo de transformaciones incentivó la realización de diferentes encuestas aplicadas en diferentes ámbitos geográficos. Básicamente, estas acciones demoscópicas buscaban conocer los cambios en los hábitos alimentarios durante los primeros meses de la pandemia, algunos de cuyos resultados ya han sido publicados.

El trabajo de Pérez Rodrigo y cols. ${ }^{9}$ refiere cambios alimentarios en el periodo de confinamiento en España con tendencia hacia un mayor consumo de alimentos saludables, menor consumo de alimentos de menos interés nutricional y aumento de la práctica de cocinar en casa. Resultados similares aparecen en el estudio llevado a cabo por Rodríguez Pérez y cols. ${ }^{10}$ al indicar que durante las primeras semanas del confinamiento los encuestados habrían aumentado el consumo de vegetales, legumbres y frutas y disminuido la ingesta de fritos, comida rápida, bebidas carbonatadas y carnes rojas.

Por su parte, el estudio llevado a cabo por los investigadores del Basque Culinary Center sobre comportamiento del consumidor en tiempos de confinamiento, elección de alimentos y actitudes culinarias en España ${ }^{11}$, pretendía determinar la percepción de los consumidores sobre sus propias elecciones y hábitos alimentarios durante el confinamiento e identificar aquellos que no resultaban adecuados. Los resultados obtenidos muestran que los hogares españoles presentaron diferentes actitudes alimentarias durante dicho período. Para los autores de la investigación, los hábitos alimentarios menos saludables podrían estar relacionados con factores emocionales como el estrés. Por el contrario, otro grupo destacado de encuestados mostraron actitudes centradas en tratar de mantener hábitos más saludables a la par que mostraban un mayor interés por cocinar y hacer deporte.

Con una aproximación más cualitativa, en el trabajo de Moraes Prata y cols. sobre los cambios que afectaron durante el confinamiento al acto de comer ${ }^{12}$, se destaca la importancia que adquirió la alimentación en la cotidianidad del confinamiento. El aumento de la actividad culinaria se habría acompañado de cambios en las prácticas y significaciones asociadas a la 
comida, al acto de comer y al de cocinar. Además de acentuarse roles de género relacionados con los cuidados, especialmente en el caso de las madres, los resultados también apuntan hacía una redefinición de las relaciones alimentación-salud-gestión corporal asociadas al acto alimentario en el sentido de una mayor indulgencia e incremento del protagonismo del placer y el bienestar. Para las autoras de la investigación se trataría de estrategias de reinvención y control asociadas a una moral del confinamiento generada por un contexto de desequilibrios e incertidumbres.

Muchos de los cambios observados en estos estudios se repiten en otros contextos como el polaco $^{13}$ o el italiano ${ }^{14,15}$. Aunque en este último caso, los autores de los estudios insisten en la provisionalidad de los resultados y en la necesidad de llevar a cabo estudios similares que ayuden a gestionar el impacto de futuras pandemias sobre los hábitos alimentarios.

En contextos como el latinoamericano, los trabajos que han analizado el impacto de la COVID-19 sobre los hábitos alimentarios han observado un descenso en el consumo de alimentos con potencial inmunomodulador como frutas y verduras y un aumento en la ingesta de golosinas, bebidas azucaradas y alcohólicas ${ }^{16}$.

Como hemos podido comprobar, se trata de investigaciones que buscaban conocer los cambios en los hábitos alimentarios o en las percepciones y significados asociados al acto de comer durante los primeros meses de la pandemia, pero que incidían menos en el análisis de las transformaciones producidas en las prácticas culinarias y gastronómicas.

Por esta razón, se consideró la conveniencia de preparar un cuestionario con el que analizar los cambios en las prácticas culinarias y gastronómicas, mostrando las diferencias antes y durante el confinamiento. Este enfoque es de especial interés teniendo en cuenta que la evaluación de la ingesta dietética también debe incluir las formas de preparación culinaria, los estilos de comer o la convivialidad $^{17}$, tal y como señaló el grupo de expertos que participó en la Jornada sobre nutrición y gastronomía en las comunidades autónomas españolas que tuvo lugar en Segovia en 2018.

Bajo estos objetivos, el presente estudio se difundió en redes sociales y medios de comunicación locales entre los días 28 de abril de 2020 y el 10 de mayo de 2020. Los resultados obtenidos y su análisis conforman el trabajo que desarrollamos a continuación.

El objetivo del estudio fue conocer los cambios producidos en las prácticas culinarias y gastronómicas durante la fase cero del estado de alarma. Como objetivos específicos, la encuesta buscaba averiguar la frecuencia y tiempo dedicado a la cocina, las motivaciones para cocinar, el tipo de cocina practicado, con quien se cocinaba y las fuentes de información utilizadas, así como la posible mejora de los conocimientos y habilidades culinarias. Otros indicadores estaban 
relacionados con el cocinado de platos tradicionales autóctonos y foráneos o internacionales. El resto de cuestiones planteadas pretendían saber si se habían producido cambios en la provisión y tipo de alimentos adquiridos y en las prácticas de convivialidad en el acto de comer. Por último, con el fin de detectar posibles cambios en las percepciones de los encuestados tras el impacto de la COVID-19, se preguntaba por el trabajo que realizan productores, agricultores, ganaderos y pescadores, por el consumo de productos ecológicos y de proximidad, por la compra en mercados y comercio local, por la alternativa que representan los huertos urbanos o la preparación propia de alimentos y por la importancia de una alimentación equilibrada, sostenible y respetuosa con el medio ambiente. 


\section{MATERIAL Y MÉTODOS}

Se ha realizado un estudio observacional y transversal aunque con enfoque diacrónico a partir de la percepción y experiencias de los entrevistados: antes/durante el confinamiento.

\section{Universo y muestra}

Muestreo no probabilístico, de conveniencia, con ajuste final a la población real por cuotas de sexo, edad y nivel de estudios, empleando coeficientes de ponderación. El universo lo compone población de 18 a 69 años residente en la provincia de Alicante. Dado que se trata de una encuesta a través de Internet, se ha decidido establecer límite máximo en 69 años porque según datos de la Encuesta sobre equipamiento y uso de tecnologías de la información y comunicación en los hogares 2019 del Instituto Nacional de Estadística ${ }^{18}$, hasta esa edad el uso de Internet se da en más del $80 \%$ de los sujetos; en cambio, a partir de los 70 disminuye considerablemente la proporción de usuarios y por tanto los internautas no representan adecuadamente al conjunto de sus propias cohortes de edad.

A nivel territorial, el foco de la investigación se puso en la provincia de Alicante, dejando disponible la encuesta al resto de provincias españolas con la intención de poder llevar a cabo comparaciones entre provincias si los datos lo permitían. Finalmente, sólo se ha obtenido una cifra adecuada en el caso de la demarcación territorial de Alicante.

\section{Secuencia del trabajo de campo}

Se diseñó un cuestionario ad hoc que se dividió en 4 partes. Mediante las distintas secciones se recogió la información sociodemográfica y la concerniente a las prácticas culinarias y gastronómicas previas y durante el confinamiento por la COVID-19.

En la sección 1 se detallaban las características del estudio, se informaba sobre quiénes eran los promotores de la investigación, en este caso la Cátedra Carmencita de Estudios del Sabor Gastronómico, una de las cátedras institucionales de la Universidad de Alicante, y GasterraCentro de Gastronomía del Mediterráneo UA-Dénia. Se indicaba la finalidad y objetivos de la misma, y la anonimización de los datos aportados. Se facilitaban datos de contacto para cualquier información adicional o consulta. Así mismo se informaba del tiempo máximo que implicaba contestar el cuestionario, tras comprobar previamente su rango temporal en un pretest de 22 casos. Tras este recorrido informativo, en la sección 2 se invitaba al entrevistado a dar su consentimiento informado para poder acceder a las siguientes secciones. La sección 3 constaba de 15 ítems de carácter sociodemográfico. La sección 4 constaba de 24 ítems con diferentes formatos de respuesta (categorización dicotómica, escala ordinal, y preguntas de respuesta abierta, tanto numéricas como alfanuméricas) relacionados con las prácticas 
culinarias y gastronómicas y la percepción sobre la producción y consumo de alimentos. La selección de los ítems se llevó a cabo entre los miembros del equipo de investigación, ampliada al juicio de expertos, entre los que contamos con académicos del área de nutrición y profesionales de la gastronomía que colaboran habitualmente con Gasterra, el centro mencionado. No se utilizó ningún cuestionario previo ni adaptado al no encontrar ninguno que incluyera cuestiones gastronómicas relacionadas con una situación excepcional de confinamiento temporal.

Se utilizó como soporte online para la recogida de información la aplicación Formularios de Google. Los entrevistados fueron reclutados a través de Internet con difusión en redes sociales y medios de comunicación locales. La recogida de información se produjo entre los días 28 de abril de 2020 y el 10 de mayo de 2020, fecha en la que se iniciaba la Fase 1 de la llamada "desescalada" en algunos territorios de la provincia de Alicante y del conjunto del Estado. Respondieron al cuestionario 1.045 personas, pero se eliminaron 5 casos que no completaron al menos el 80\% del cuestionario, 21 casos de respuestas duplicadas, 2 casos de respuestas incongruentes, 42 casos mayores de 69 años y, finalmente, los 296 casos de personas residentes en otras provincias. Por tanto, finalmente la muestra operativa fue de 679 casos.

\section{Aspectos deontológicos}

El protocolo de investigación se llevó a cabo de acuerdo con la declaración de Helsinki para estudios en humanos de la Asociación Médica Mundial, con estricto respeto a la confidencialidad de la información de acuerdo a la Ley Orgánica 15/1999 de 13 de diciembre de protección de datos de carácter personal en todos los procesos de recogida y tratamiento de la información obtenida y La Ley Orgánica 3/2018 de 5 de diciembre de Protección de Datos Personales y garantía de los derechos digitales. El presente estudio ha sido aprobado por el Comité de Ética de la Universidad de Alicante.

\section{Análisis estadístico}

El análisis de los datos se ha realizado utilizando como soporte el paquete estadístico IBM SPSS Statistics 25. Una parte importante del análisis ha consistido en la comparación entre los hábitos previos al confinamiento por el COVID-19 y los hábitos durante ese período excepcional. Aunque estrictamente se trata de un estudio observacional, en cierta medida sería una aproximación al pre-experimento pre-test/post-test de un grupo ${ }^{19}$, con la diferencia de que en este caso no se realizan tomas de información en tiempos distintos, sino que se realiza una única toma de información referida a dos tiempos, antes y durante el confinamiento, que son los que se comparan. Lógicamente, la situación (pre)experimental es el confinamiento, que ha despertado 
enorme interés académico por su excepcionalidad y por constituir una situación que, en una u otra medida, ha afectado a la gran mayoría de la población total o parcialmente. Para afrontar estadísticamente las diferencias entre un tiempo y otro, se ha asumido que se trata de muestras relacionadas: se pregunta lo mismo para ambos tiempos con los mismos indicadores y a las mismas personas y, por tanto, cada par de indicadores tiene una alta probabilidad de estar asociado. Por tanto, para identificar si las diferencias entre un tiempo y otro son estadísticamente significativas se debe considerar el supuesto de muestras emparejadas o relacionadas, y a partir de ello se ha utilizado la prueba de suma de rangos de Wilcoxon para variables ordinales y el test de McNemar para variables nominales, en ambos casos, se han considerado diferencias significativas cuando los niveles de significación eran inferiores a 0,05, como ya han hecho anteriormente Coll-Planas y cols. ${ }^{20}$.

Para el resto de indicadores no emparejados, que normalmente comparan en el enunciado la situación de confinamiento con la previa (por ejemplo, “¿Está Ud. cocinando más durante el confinamiento [que anteriormente]?"), se ha realizado un análisis descriptivo. Con los indicadores actitudinales se ha realizado un análisis factorial con rotación Varimax para ver si correlacionan de manera conjunta. El análisis factorial ha sido pertinente al obtener una medida de adecuación $\mathrm{KMO}=0,785$ y una significación del test de Bartlett $<0,001$, además de la correlación de anti-imagen en todos los casos por encima de 0,7.

También se han contemplado relaciones con variables sociodemográficas: sexo (49,6\% hombres, $50,4 \%$ mujeres); edad, tratada en estadísticos como una variable cuantitativa, pero en las tablas expresada como una escala ordinal adecuada a los intervalos de ponderación: 18-34 (24,5\%), 3554 (47,4\%), 55-69 (28,1\%); nivel de estudios, presentada como una escala de 3 categorías: primarios o inferior $(10,1 \%)$, secundarios $(53,4 \%)$ y universitarios $(36,5 \%)$; ámbito rural $(26,3 \%)$ o urbano (73,7\%); estado civil, distribuido en casado-a (52,3\%), soltero-a (18,7\%), pareja de hecho o en convivencia (18,8\%), divorciado-a/separado-a (7,2\%) y viudo-a (2,2\%); situación laboral, que recoge las categorías estudiante (8,9\%), asalariado (60,8\%), autónomo/empresario (18\%), pensionista $(5,6 \%)$ y desempleado $(6,7 \%)$; ingresos en el hogar, se sintetizo en bajos $(21,6 \%)$, medios $(72,4 \%)$ y altos (6\%). Aquellas categorías que no recogen un número de casos suficiente (viudo-a, otras situaciones laborales...) no se incluyen en las tablas de contingencia. Se han calculado estadísticos de asociación Phi, Eta o Lambda según correspondiese. 


\section{RESULTADOS}

\section{Comparación de hábitos culinarios y gastronómicos antes y durante el confinamiento}

Durante el confinamiento aumentaron tanto la frecuencia con la que se cocinaba como el tiempo dedicado a cocinar (Tabla 1, a y b), tal como declaró más del 70 \% de los entrevistados. De hecho, un parámetro como la mediana, poco sensible a variaciones en escalas ordinales cortas, aumenta en las dos primeras variables de la Tabla 1, pasando de 2 ("algunos días a la semana") a 3 ("a diario") en frecuencia, y de 1 ("menos de 1 h/día") a 2 ("entre 1 y 3 h/día) en tiempo que dedica a cocinar. La disponibilidad de tiempo (82\%) y su menor sujeción a calendarios rígidos $(44,4 \%)$ permite esa notable variación de acuerdo con las principales motivaciones para incrementar la práctica culinaria que manifiestan los entrevistados (ver Figura 1). Otro tipo de argumentos como la posibilidad de cocinar en familia, la posibilidad de hacer platos nuevos o el aburrimiento, quedan en un segundo plano con proporciones por debajo del $30 \%$ y el resto de categorías no superan el $10 \%$. 
Tabla 1. Prácticas culinarias antes y durante el confinamiento por el COVID-19 (\%).

\begin{tabular}{|c|c|c|}
\hline & Antes & Durante \\
\hline \multicolumn{3}{|l|}{ Frecuencia con la que cocina (a). } \\
\hline Nunca & 5,7 & 3,9 \\
\hline Los fines de semana & 12,2 & 3,8 \\
\hline Algunos días a la semana & 36,6 & 23,3 \\
\hline A diario & 45,7 & 69,1 \\
\hline Total & 100,0 & 100,0 \\
\hline Mediana & 2 & 3 \\
\hline \multicolumn{3}{|l|}{ Tiempo que dedica a cocinar (b). } \\
\hline No cocinaba & 5,8 & 3,5 \\
\hline Menos de 1 hora/día & 52,4 & 20,9 \\
\hline Entre 1 y 3 horas/día & 35,5 & 63,5 \\
\hline Más de 3 horas al día & 6,3 & 12,2 \\
\hline Total & 100,0 & 100,0 \\
\hline Mediana & 1 & 2 \\
\hline \multicolumn{3}{|l|}{ Fuentes de aprendizaje en la cocina (c). } \\
\hline Entorno familia & 80,6 & 53,7 \\
\hline Libros, recetarios, blogs, TV, redes & 36,8 & 58,1 \\
\hline Curso presencial de cocina & 8,4 & 6,1 \\
\hline Curso online de cocina & 2,9 & 4,2 \\
\hline \multicolumn{3}{|l|}{ Tipo de cocina (d). } \\
\hline Tradicional & 79,7 & 85,6 \\
\hline De improvisación & 29,0 & 28,9 \\
\hline De precocinados & 10,1 & 4,6 \\
\hline Otros & 3,8 & 4,9 \\
\hline \multicolumn{3}{|l|}{ Tipos de platos que cocina (e). } \\
\hline Platos principales & 87,2 & 90,4 \\
\hline
\end{tabular}




\begin{tabular}{|l|c|c|}
\hline Aperitivos & 32,1 & 34,2 \\
\hline Postres & 20,8 & 35,5 \\
\hline Repostería salada & 7,7 & 14,5 \\
\hline Con quién cocina (f) & 74,6 & 59,3 \\
\hline Solo & 26,5 & 37,5 \\
\hline Pareja & 7,7 & 18,6 \\
\hline Hijos/as & 8,9 & 9,2 \\
\hline Madre & 3,4 & 3,2 \\
\hline Padre & 1,9 & 2,1 \\
\hline Otros & & \\
\hline
\end{tabular}

(a) Escala: 0=nunca, 1=fines de semana, 2=algunos días de la semana, 3=a diario. Significación asintótica bilateral $=0,0000001$ en la prueba de suma de rangos de Wilcoxon.

(b) Escala: $0=$ no cocinaba, 1=menos de $1 \mathrm{hr}$./día, 2=entre 1 y $3 \mathrm{hrs} . /$ día, 3=más de $3 \mathrm{hrs}$./día). Significación asintótica bilateral=0,0000001 en la prueba de suma de rangos de Wilcoxon. (c) La suma de los porcentajes no es igual a 100, ya que la pregunta es multirrespuesta. Significaciones exactas bilaterales en el test de McNemar en cada categoría (tomadas como variables dicotómicas): Entorno familiar $=0,0000001$, Libros, recetarios... $=0,0000001$, Curso presencial de cocina $=0,009$, Curso online de cocina $=0,075$.

(d) La suma de los porcentajes no es igual a 100, ya que la pregunta es multirrespuesta. Significaciones exactas bilaterales en el test de McNemar en cada categoría (tomadas como variables dicotómicas): Tradicional=0,000037, De improvisación $=1$, De precocinados $=0,000002$, Otros=0,096.

(e) La suma de los porcentajes no es igual a 100, ya que la pregunta es multirrespuesta. Significaciones exactas bilaterales en el test de McNemar en cada categoría (tomadas como variables dicotómicas): Platos principales $=0,012$, Aperitivos $=0,198$, Postres $=0,0000001$, Repostería salada $=0,0000001$.

(f) La suma de los porcentajes no es igual a 100, ya que la pregunta es multirrespuesta. Significaciones exactas bilaterales en el test de McNemar en cada categoría (tomadas como variables dicotómicas): Solo=0,0000001, Pareja =0,0000001, Hijos/as=0,0000001, Madre=0,851, Padre $=1$, Otros $=0,804$. 
Figura 1. Motivos por los que ha cocinado más durante el confiamiento.

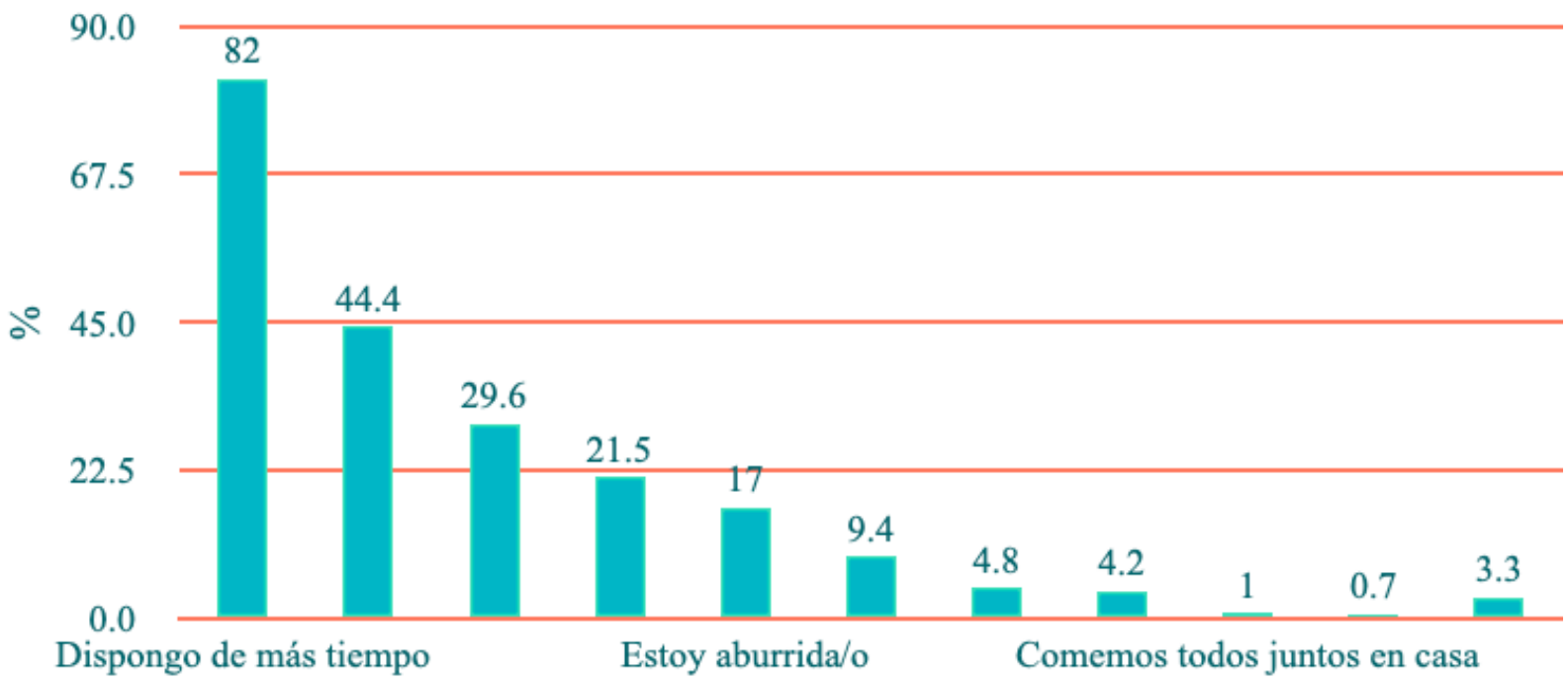

Nota: la suma de los porcentajes no es igual a 100, ya que la pregunta es multirespuesta. Base: 507 encuestados que cocinan más 
Las diferencias entre los hábitos previos al estado de alarma y los comportamientos que han tomado forma durante el confinamiento son estadísticamente significativas, tanto en frecuencia de cocinado generalizado durante el confinamiento como en tiempo que dedica a cocinar, tal como se puede observar con unos niveles de significación prácticamente residuales y que, por tanto, dejan poco margen a la duda.

Cocinar "a diario", que no era un hábito mayoritario antes del confinamiento (45,7\%), pasó a ser un comportamiento muy generalizado durante el confinamiento (cerca del 70\%). La mayoría $(52,4 \%)$ cocinaba menos de una hora antes del confinamiento pero con el estado de alarma y las medidas de restricción de movimientos que conllevaba, cerca de dos tercios de la población alicantina entrevistada pasó a cocinar entre 1 y 3 horas al día. En el caso de los cocinados largos, "más de 3 horas", se pasó de un 6,3\% a un 12,2\%.

En el resto de indicadores de la Tabla 1 que dan cuenta de cómo se concretaba el cocinado (fuentes de aprendizaje, tipo de cocina, tipo de platos y socialización), obtenido el test de McNemar para todas las categorías (tomadas como variables dicotómicas), las diferencias entre 'antes' y 'durante' son estadísticamente significativas (significación exacta bilateral<0,05) en todos los casos excepto en 'Curso online de cocina' (c), 'De improvisación' y 'Otro tipo de cocina' (d), 'Aperitivos' (e), y 'Madre', 'Padre' y 'Otros' (f). Por tanto, en general se han producido cambios significativos al sobrevenir la situación de confinamiento.

Respecto al primero de estos indicadores, las fuentes de aprendizaje en la cocina (Tabla 1.c), el entorno familiar, en concreto la transmisión intergeneracional de saberes culinarios, dominaba con claridad antes del confinamiento a otro tipo de fuentes: $80,6 \%$ frente a $36,8 \%$ en el caso de libros, recetarios, blogs, webs, TV, etc. Una situación que cambió durante el confinamiento al pasar estas últimas al 58,1\%. No obstante, la transmisión de saberes desde el entorno familiar continuó estando presente en la mayoría de los casos $(53,7 \%)$.

\section{Tipo de cocinado}

Las diferencias en el tipo de cocina desarrollado antes y durante el confinamiento son estadísticamente significativas (Tabla 1.d). En ambos momentos domina con claridad la cocina "tradicional", pero ésta experimenta una ligera variación al alza durante el confinamiento (alrededor de 6 puntos porcentuales) en detrimento de los precocinados, cuyo porcentaje se ha visto reducido en más de la mitad.

Los resultados también muestran que no solo se ha cocinado más sino también más variado (Tabla 1.e). Los cambios más sustanciales se dan en "postres" (de 20,8\% antes del confinamiento a 35,5\% durante) y "repostería salada" (de 7,7\% a 14,5\%). 


\section{Cocina y sociabilidad}

Como muestran los resultados (Tabla 1.f), se ha incrementado el cocinado en compañía: el cocinado solo desciende en más de 15 puntos durante el confinamiento, mientras que aumentan todas las categorías de elaboración grupal, particularmente "pareja" e "hijos/as" (alrededor de 11 puntos porcentuales en ambos casos).

Como se puede comprobar en los resultados reflejados en la tabla 2 , el evento que en mayor medida se celebraba ya en compañía antes del confinamiento, la cena (más del 70\% cenaba acompañado), es el que menos ha variado y el único en que no se registran diferencias significativas, como se puede apreciar con las significaciones exactas bilaterales del test de McNemar. Los cambios se producen en el resto de comidas, siempre en el sentido de incrementar las situaciones de ingesta en compañía debido a la convivencia forzosa del confinamiento. Respecto a realizar otras actividades los incrementos de las comidas en compañía son siempre mayores cuando se incluye la televisión, y los decrementos de las comidas "solo/a" son menores cuando se están haciendo otras cosas. Por tanto, se ha comido en mayor medida en compañía pero también con mayor presencia de dispositivos audiovisuales. 
Tabla 2. Con quién se compartían las diferentes comidas del día antes y durante el confinamiento por COVID-19 (\% válido para cada comida).

\begin{tabular}{|c|c|c|}
\hline & Antes & Durante \\
\hline \multicolumn{3}{|l|}{ Desayuno } \\
\hline Solo/a & 61,0 & 44,4 \\
\hline Solo/a haciendo otras cosas & 16,8 & 17,9 \\
\hline En compañía + TV & 5,5 & 13,9 \\
\hline En compañía sin TV & 11,2 & 19,3 \\
\hline No realizaba esta comida & 5,5 & 4,5 \\
\hline Total & 100,0 & 100,0 \\
\hline \multicolumn{3}{|l|}{ Almuerzo } \\
\hline Solo/a & 36,5 & 27,7 \\
\hline Solo/a haciendo otras cosas & 14,6 & 15,8 \\
\hline En compañía + TV & 9,1 & 13,3 \\
\hline En compañía sin TV & 20,6 & 16,8 \\
\hline No realizaba esta comida & 19,2 & 26,3 \\
\hline Total & 100,0 & 100,0 \\
\hline \multicolumn{3}{|l|}{ Comida } \\
\hline Solo/a & 15,7 & 8,1 \\
\hline Solo/a haciendo otras cosas & 18,8 & 15,0 \\
\hline En compañía + TV & 40,3 & 49,7 \\
\hline En compañía sin TV & 23,2 & 27,0 \\
\hline No realizaba esta comida & 1,9 & 0,2 \\
\hline Total & 100,0 & 100,0 \\
\hline \multicolumn{3}{|l|}{ Merienda } \\
\hline Solo/a & 35,2 & 23,7 \\
\hline Solo/a haciendo otras cosas & 14,8 & 16,5 \\
\hline En compañía + TV & 6,6 & 17,4 \\
\hline
\end{tabular}




\begin{tabular}{|l|c|c|}
\hline En compañía sin TV & 11,2 & 16,3 \\
\hline No realizaba esta comida & 32,3 & 26,1 \\
\hline Total & 100,0 & 100,0 \\
\hline Cena & 11,5 & 9,9 \\
\hline Solo/a & 15,6 & 14,2 \\
\hline Solo/a haciendo otras cosas & 51,0 & 53,7 \\
\hline En compañía + TV & 20,4 & 20,6 \\
\hline En compañía sin TV & 1,3 & 1,7 \\
\hline No realizaba esta comida & 100,0 & 100,0 \\
\hline Total & \multicolumn{2}{|c|}{} \\
\hline $\begin{array}{l}\text { Nota: significaciones exactas bilaterales en el test de McNemar: Desayuno=0,0000001, } \\
\text { Almuerzo=0,0000001, Comida=0,0000001, Merienda=0,0000001, Cena=0,188. }\end{array}$ \\
\hline
\end{tabular}


El almuerzo y/o la merienda son, tanto en confinamiento como en situación de normalidad, las que se suele saltar una proporción mayor de población. El cambio que ha implicado el confinamiento es que aumenta el porcentaje de entrevistados que ha obviado el almuerzo en confinamiento, pero desciende la cifra relativa de quienes se han saltado la merienda.

\section{Habilidades culinarias}

Aunque hay un relativamente elevado porcentaje de entrevistados (18,3\%) que no ha percibido ninguna mejora, la gran mayoría, prácticamente dos tercios, declara una mejora media o elevada en sus habilidades culinarias a raíz del confinamiento (de 5 a 10) e incluso un $32 \%$ se sitúa entre 7 y 10 , que implica un nivel de mejora muy destacado (Figura 3). 
Figura 3. Autopercepción de mejoras en las habilidades culinarias.

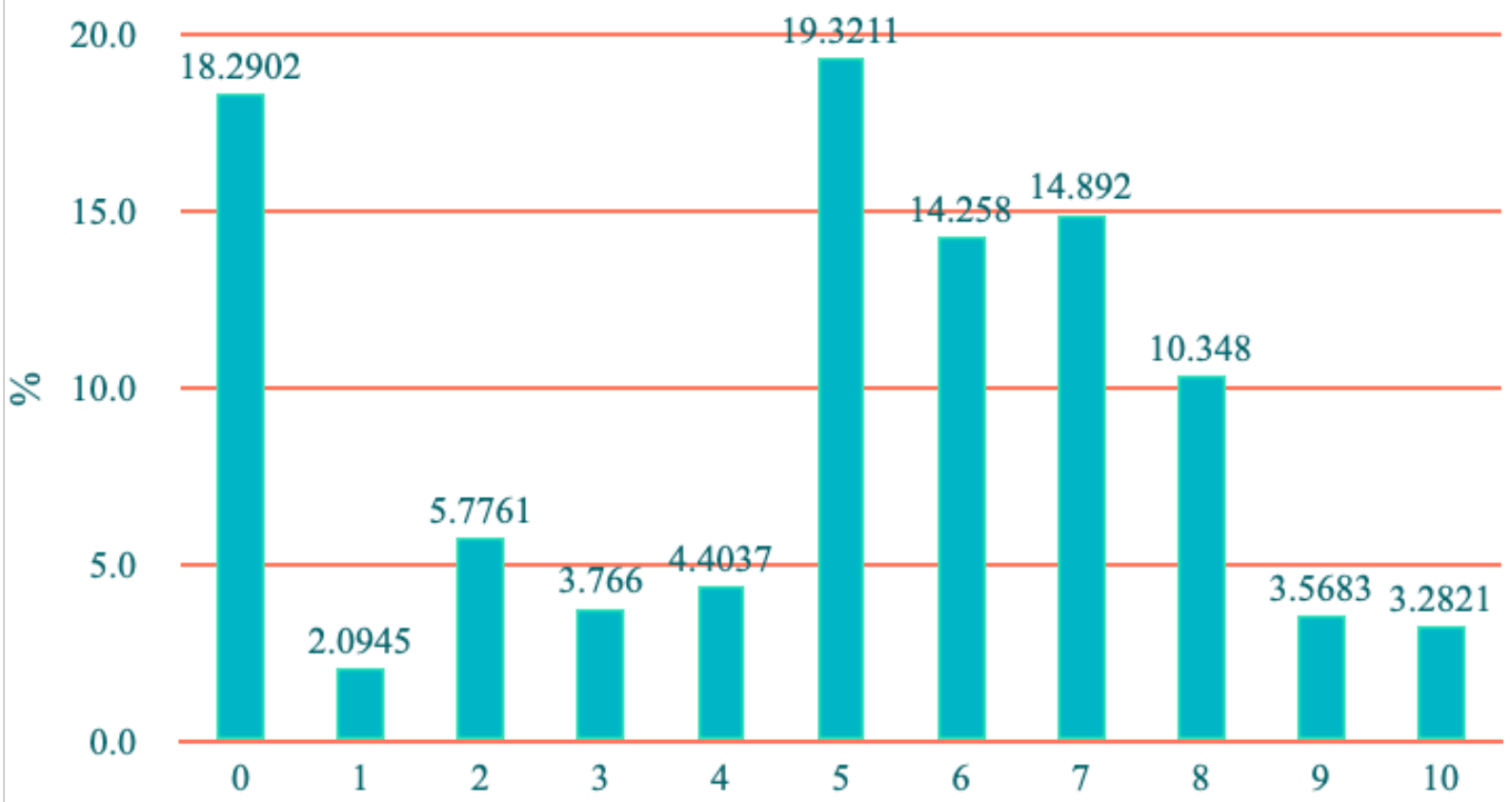

Escala de 0 a 10, donde 0 es ninguna mejora y 10 es máximo grado de mejora. Media $=4,77$; desviación típica=2,94 (asumiendo el supuesto de una escala de intervalo); mediana $=5$. 
La población alicantina se ha decantado sobre todo por explorar su propia cocina tradicional, la de sus comarcas y ampliado al resto de la comunidad autónoma: más del $60 \%$ ha hecho por primera vez en el confinamiento algún plato valenciano que no había hecho anteriormente. También destaca que más de la tercera parte se ha atrevido con elaboraciones de cocina internacional y el $17,1 \%$ con platos tradicionales de otras comunidades autónomas.

Respecto a los platos tradicionales valencianos (ver Figura 4), tres son los grupos que destacan por encima del resto con alrededor de un tercio de los casos válidos: platos de cuchara, con amplia variedad, con un predominio de las variantes autóctonas del cocido, las lentejas y la olleta, paella y otros arroces; y postres, con protagonismo para las monas de Pascua por la época en que se produjo el confinamiento. "Pan y otras masas saladas", especialmente cocas de base de tomate, y "otros guisos" también registran porcentajes destacados, en torno al 25\%. Asimismo, es necesario mencionar una categoría, "pastas tradicionales", que registra un porcentaje menor, pero que surge fundamentalmente de dos platos, la fideuà y el gazpacho de la Foia de Castalla o el Vinalopó, comarcas próximas a la Mancha. 
Figura 4. Platos tradicionales locales/regionales que ha realizado por primera vez durante el confinamiento.

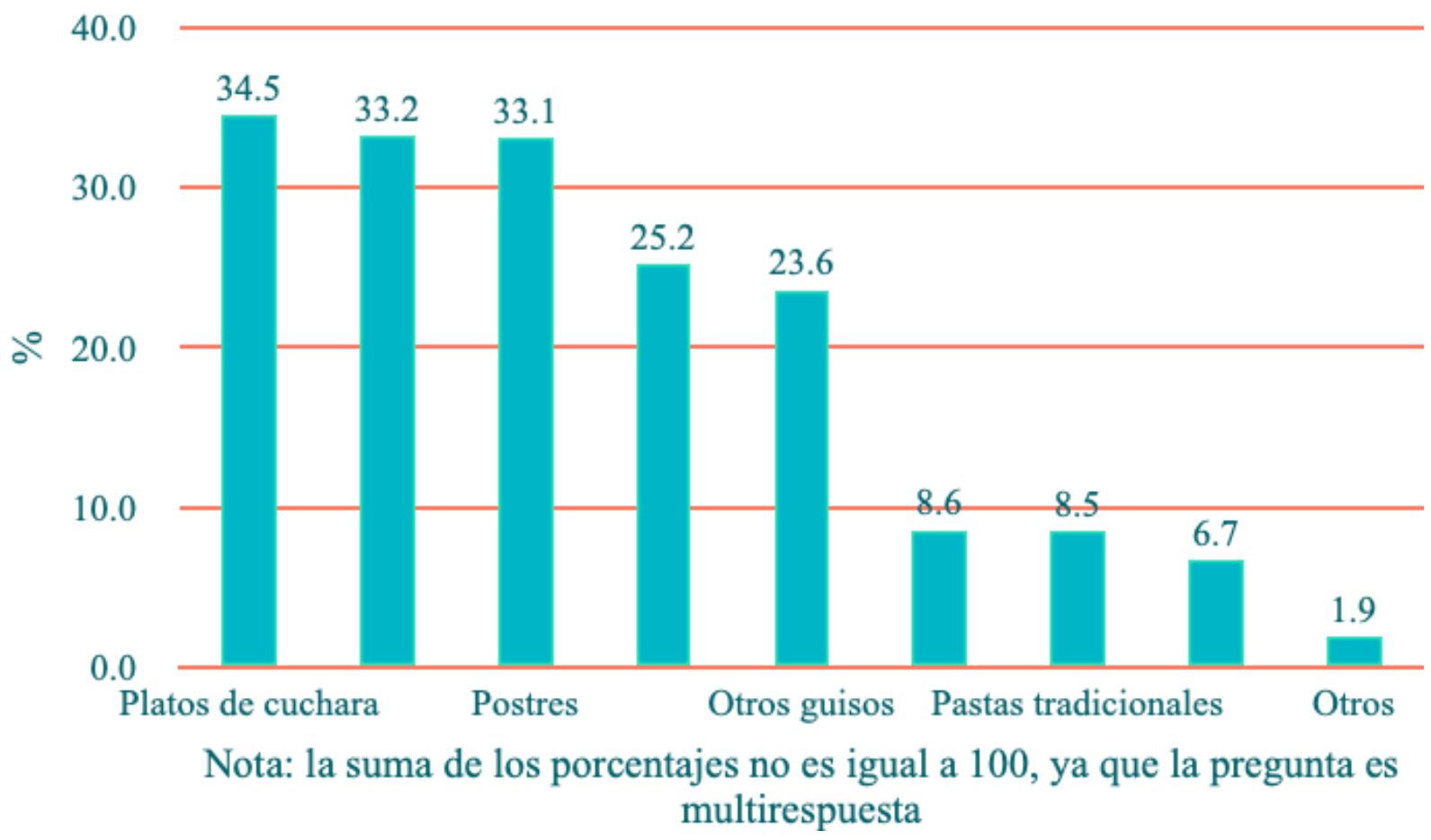


En cuanto a las elaboraciones de otras comunidades autónomas (Figura 5), la población alicantina ha cocinado platos de tres territorios claramente por encima del resto: Asturias, País Vasco y Castilla-La Mancha. De hecho, en este último caso, algunos de sus platos pasan a ser considerados platos autóctonos, sobre todo en las comarcas alicantinas que lindan con esta última comunidad autónoma. 
Figura 5. Comunidad Autónoma de Origen de las Recetas

40.0

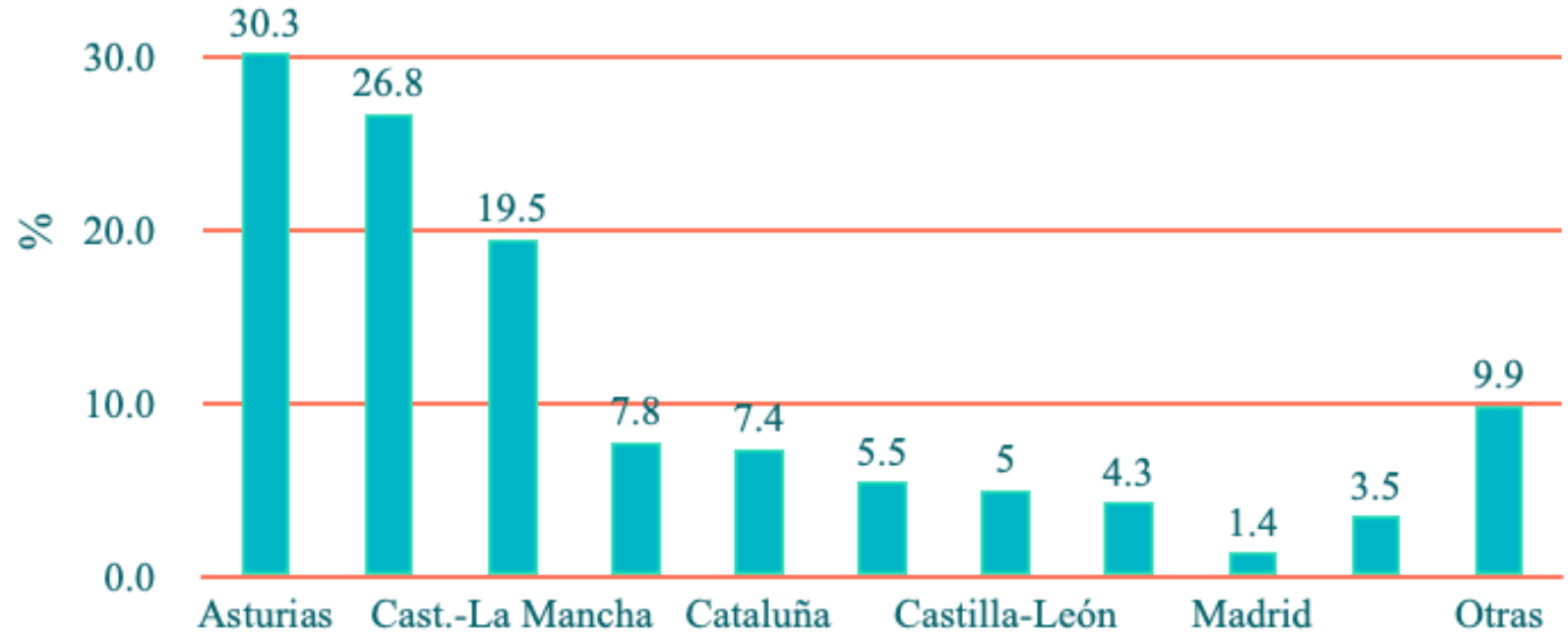

Nota: la suma de los porcentajes no es igual a 100, ya que la pregunta es multirespuesta 
Finalmente, en los platos internacionales (Figura 6), Francia, icónico referente de la cocina mundial, queda relegado a posiciones de menor jerarquía en las cocinas alicantinas en favor de platos de otras latitudes, que por lo general requieren menos elaboración e incluso en algunos casos podrían ser encuadrados en la categoría fast food. Así, Italia ocupa la primera posición sobre todo gracias a las pizzas y diferentes tipos de pasta, aunque también risottos; y México debe su lugar de privilegio casi en exclusiva a su comida rápida, que incluye burritos, fajitas, nachos, guacamole, etc. En el caso de los países árabes, tercer grupo, dominan las comidas más elaboradas: cous-cous, tajine, taboulé, aunque también los falafels o el hummus. Los platos de la India son en su mayoría los que se elaboran a partir de las salsas masala y curry, especialmente pollo al curry. La inclinación por lo oriental también queda marcada por la presencia notable de platos chinos, japoneses o de influencia asiática indeterminada o de estados del sudeste como Tailandia. 
Figura 6. País/Región de las recetas internacionales elaboradas durante el confinamiento.

40.0

33.8

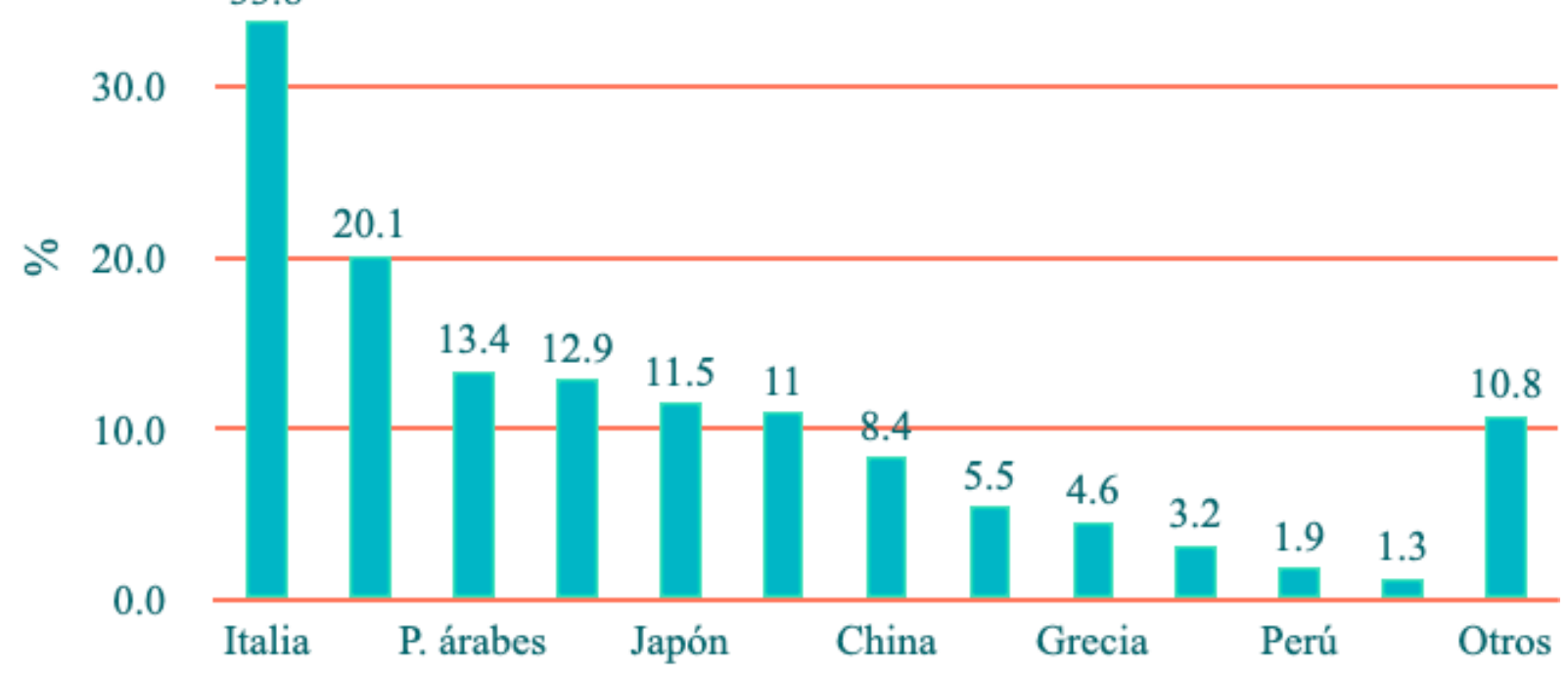

Nota: la suma de los porcentajes no es igual a 100, ya que la pregunta es multirespuesta 


\section{Cambios en la percepción del proceso de alimentación}

La pandemia, el confinamiento y sus efectos sobre el sistema alimentario (producción, distribución y consumo) ha hecho que la gran mayoría de la población alicantina entrevistada (84\%) modifique su percepción sobre alguna cuestión relacionada con la alimentación. El que ha alcanzado una proporción mayor ha sido el trabajo de los productores del sector primario $(58,1 \%)$, respecto a los cuales se ha tomado conciencia de su importancia y necesidad de valoración económica. Recordemos que el confinamiento comienza después de semanas de movilizaciones de los agricultores. La necesidad de comprar en mayor medida en mercados y comercios de proximidad y, por ende, menos en grandes-medianas superficies, además de la importancia de una alimentación equilibrada, están en torno al 50\%. Y con cerca del $40 \%$ la necesidad de cuidar el medio ambiente y consumir productos agroecológicos.

\section{El perfil de las personas encuestadas que cocinan platos tradicionales}

Finalmente, en las tablas 3 y 4 desplegamos los resultados en función de diferentes variables sociodemográficas que ayuden a entender el comportamiento de la población alicantina:

Aunque las variables sociodemográficas se asocian con dificultad a dos variables dicotómicas relevantes de este estudio, si el entrevistado ha cocinado más durante el confinamiento, y si ha realizado en ese período platos o recetas tradicionales que no había hecho nunca, parece que jóvenes, mujeres, con nivel de estudio bajo o alto, parejas no casadas y autónomos son, por lo general, los perfiles que ofrecen cifras más elevadas en los dos indicadores. Además, también se detecta que la población del medio rural se ha atrevido más con platos tradicionales que no había cocinado antes; y quienes han cocinado más que de costumbre son quienes cuentan con ingresos medios o altos. 
Tabla 3. Incremento de la frecuencia y tiempo de cocinado (\% válido sobre el total de cada categoría).

\begin{tabular}{|c|c|c|}
\hline \multirow[t]{2}{*}{ Sexo } & Masculino & $67,1 \%$ \\
\hline & Femenino & $74,6 \%$ \\
\hline \multirow[t]{3}{*}{ Edad } & 18 a 34 años & $74,9 \%$ \\
\hline & 35 a 54 años & $69,6 \%$ \\
\hline & 55 años y más & $69,6 \%$ \\
\hline \multirow[t]{3}{*}{ Nivel de estudio } & Primarios o inferior & $72,1 \%$ \\
\hline & Secundarios & $66,9 \%$ \\
\hline & Universitarios & $76,5 \%$ \\
\hline \multirow[t]{2}{*}{ Entorno } & Rural & $69,7 \%$ \\
\hline & Urbano & $71,3 \%$ \\
\hline \multirow[t]{4}{*}{ Estado civil } & Casado/a & $70,4 \%$ \\
\hline & Divorciado/a o Separado/a & $60,4 \%$ \\
\hline & En pareja & $84,5 \%$ \\
\hline & Soltero/a & $63,8 \%$ \\
\hline \multirow[t]{5}{*}{ Situación sociolaboral } & Empresario/a- Autónomo/a & $77,0 \%$ \\
\hline & Desempleado/a & $54,3 \%$ \\
\hline & Asalariado/a & $73,6 \%$ \\
\hline & Estudiante & $75,0 \%$ \\
\hline & Pensionista & $34,2 \%$ \\
\hline \multirow[t]{3}{*}{ Ingresos } & Bajos & $58,9 \%$ \\
\hline & Medios & $74,0 \%$ \\
\hline & Altos & $75,6 \%$ \\
\hline
\end{tabular}


Tabla 4. Realización durante el confinamiento de platos o recetas tradicionales que no había hecho nunca en función de diferentes variables sociodemográficas (\% válido sobre el total de cada categoría).

\begin{tabular}{|c|c|c|}
\hline \multirow[t]{2}{*}{ Sexo } & Masculino & $55,8 \%$ \\
\hline & Femenino & $69,3 \%$ \\
\hline \multirow[t]{3}{*}{ Edad } & 18 a 34 años & $68,1 \%$ \\
\hline & 35 a 54 años & $59,9 \%$ \\
\hline & 55 años y más & $62,3 \%$ \\
\hline \multirow[t]{3}{*}{ Nivel de estudio } & Primarios o inferior & $71,0 \%$ \\
\hline & Secundarios & $59,4 \%$ \\
\hline & Universitarios & $65,2 \%$ \\
\hline \multirow[t]{2}{*}{ Entorno } & Rural & $69,3 \%$ \\
\hline & Urbano & $60,2 \%$ \\
\hline \multirow[t]{4}{*}{ Estado civil } & Casado/a & $60,6 \%$ \\
\hline & Divorciado/a o Separado/a & $61,2 \%$ \\
\hline & En pareja & $67,0 \%$ \\
\hline & Soltero/a & $60,6 \%$ \\
\hline \multirow[t]{5}{*}{ Situación sociolaboral } & Empresario/a- Autónomo/a & $66,4 \%$ \\
\hline & Desempleado/a & $64,4 \%$ \\
\hline & Asalariado/a & $62,7 \%$ \\
\hline & Estudiante & $60,0 \%$ \\
\hline & Pensionista & $51,3 \%$ \\
\hline \multirow[t]{3}{*}{ Ingresos } & Bajos & $64,6 \%$ \\
\hline & Medios & $62,2 \%$ \\
\hline & Altos & $61,0 \%$ \\
\hline \multicolumn{3}{|c|}{$\begin{array}{l}\text { Para la relación con la Edad, Eta=0,333; para la relación con el Sexo, Phi=-0,14; para la } \\
\text { relación con Entorno, Phi }=-0,1 \text {. Los valores de los estadísticos para el resto de relaciones entre } \\
\text { variables (Lambda o Phi, según corresponda) revelan asociaciones "despreciables". }\end{array}$} \\
\hline
\end{tabular}




\section{DISCUSIÓN}

Los resultados constatan un aumento en la frecuencia y el tiempo dedicado a la cocina y ponen de manifiesto la importancia que adquirió la alimentación en la cotidianidad del confinamiento ${ }^{12}$. Mientras antes del confinamiento más del 50\% manifestaba cocinar menos de una hora al día, con el estado de alarma y las medidas de restricción de movimientos, cerca de dos tercios de las personas que respondieron a la encuesta pasaron a cocinar entre 1 y 3 horas al día, y cambiaron las comidas rápidas y poco elaboradas, a las que se recurre normalmente por falta de tiempo aunque también por comodidad y asunción de una pauta de elaboración e ingesta de alimentos conectada al patrón global fast food ${ }^{21}$, por platos más tradicionales. La constatación de esta actitud positiva hacia la forma de alimentarnos que acompaña a nuestras tradiciones culinarias, como ha ocurrido en el caso que nos ocupa con los platos de cuchara y los arroces, debería de tomarse en consideración en el momento de diseñar estrategias orientadas a la promoción de la dieta mediterránea 22 .

Como han señalado diversos estudios, a lo largo de las últimas décadas ha tenido lugar un progresivo abandono de los hábitos alimentarios propios de la dieta y la cultura alimentaria mediterránea por parte de la población española ${ }^{23}$. Estos mismos trabajos, al analizar los factores que han conducido a esta situación, destacan las nuevas formas de comer que se ven reflejadas tanto en los alimentos que ingerimos y su frecuencia, como en su forma de elaboración y consumo, incluyendo una menor convivialidad. Para corregir esta situación se han apuntado cambios $^{23}$ como los que se han producido en los hábitos alimentarios y gastronómicos de los alicantinos y alicantinas durante el confinamiento.

Aunque la disponibilidad de tiempo fue el principal motivo que llevó a los encuestados a cocinar más, también fue importante el poder cocinar en familia. La necesidad de involucrar a todo el núcleo familiar, y en particular a los más pequeños, en la preparación de las comidas ${ }^{24-26}$ se está reivindicando como una alternativa de educación alimentaria que puede ayudar a mejorar los hábitos alimentarios de la población. Profundizar en la motivación que ha conducido a cocinar en familia puede ser, como ocurre con la disponibilidad de más tiempo para cocinar y hacerlo optando por la cocina tradicional, otra de las cuestiones que habría que potenciar. Se trata de propuestas que también han sido recogidas en otros estudios sobre el impacto del confinamiento en los hábitos alimentarios ${ }^{15}$. La incorporación de la actividad culinaria al bagaje de actividades en común de la familia permitió resituar el conocimiento y la experiencia culinaria entre los elementos que forman parte de la interacción en nuestra fuente de socialización primaria. Otra cuestión es el tipo de cocina y de alimentación que se refuerce en esta interrelación, pero al 
menos el confinamiento condujo a que este campo de la actividad reproductiva recobrase protagonismo en un momento donde las familias no tienen la función "totalizadora" de antaño ${ }^{27}$. Aunque los resultados muestran un incremento de la convivialidad en el acto de comer durante el confinamiento, la investigación también ha puesto de manifiesto comportamientos menos positivos, como la presencia de dispositivos audiovisuales como el móvil, el ordenador o la televisión mientras se comparte la comida. Estos hábitos han persistido e incluso se han incrementado en algunos casos. No olvidemos que tan importante es lo que comemos como el cómo lo comemos y con quién ${ }^{28}$.

Así mismo, las personas encuestadas respondieron haber preparado más postres y más recetas de repostería salada durante el confinamiento. Dicho resultado está en sintonía con los incrementos en la compra de harina reportados por el Ministerio de Agricultura, Pesca y Alimentación para la duodécima semana del año, llegando incluso a momentos puntuales de desabastecimiento. El consumo de este producto fue un 196\% mayor que en la misma semana del año anterior, muy por encima del siguiente grupo de alimentos en cuanto a crecimiento, las legumbres, que se compraron un $68 \%$ más. También hay que destacar el incremento del $50 \%$ en la compra de azúcar, un ingrediente central en la elaboración de postres ${ }^{8}$. El componente lúdico del amasado y las decoraciones de postres en un momento en el que hay tiempo y afán de evasión puede explicar la atención dedicada a estas recetas, pero tampoco se debe desdeñar como factor explicativo, la inclinación por platos más calóricos en tiempos o momentos de crisis y por tanto de mayor estrés, tal como demostraron en su experimento durante la crisis económica de 2008 Laran \& Salerno ${ }^{29}$, como parte de un mecanismo innato de previsión/adaptación que explica la teoría de la historia de vida ${ }^{29}$ o como han mostrado otros estudios referidos a cambios alimentarios durante el confinamiento por COVID-19 12 .

En relación con los cambios en el tiempo dedicado a la cocina y las recetas que se prepararon durante el confinamiento, aunque éste no contribuyó a asentar la pauta de cinco comidas que ya cumplía una ajustada mayoría de la población de la provincia de Alicante, sí que las reconfiguró discretamente, en línea con lo que han señalado otros estudios que se han ocupado de las consecuencias y el impacto de la COVID-19 sobre determinados hábitos ${ }^{30}$. Además de realizar desayunos más tardíos y copiosos, que acabaron retrayendo la voluntad/necesidad de un almuerzo a media mañana, la presencia de toda la familia en casa por la tarde y en algunos casos cocinando dulces, fue lo que permitió aumentar la proporción de población que ha merendado regularmente durante el confinamiento. El estado de alarma coincidió con la época 
de la tradicional "mona de Pascua", que muchas personas se atrevieron a preparar; al igual que ocurrió con las toñas, las cocas, o las magdalenas.

En relación con las fuentes de información utilizadas para cocinar durante el confinamiento destacó el mayor uso de internet ${ }^{31}$ aunque persistió el recurso a la trasmisión de saberes desde el entorno familiar. El incremento del protagonismo de la red global debe hacernos reflexionar acerca de la calidad de la información que ofrecen muchos de los blogs, recetarios online, etc. ${ }^{32-34}$, ya que se trata de una cuestión relevante por su influencia en la configuración de las modas y las tendencias culinarias, alimentarias y gastronómicas ${ }^{35}$. Sin olvidar el papel que juegan las redes sociales, que vieron incrementado su uso durante el confinamiento, a la hora de viralizar determinados tipo de comportamientos, incluidos los culinarios ${ }^{36,37}$.

En consonancia con la importancia que adquirió el acto de cocinar, los resultados muestran una autopercepción de mejora de las habilidades culinarias, a pesar de que una parte de las personas haya asumido la ausencia de progresos, en buena medida porque son un segmento que apenas cocinó o se limitó a elaborar los platos de siempre; se trata sobre todo de población mayor y de menor nivel de estudios.

Durante el confinamiento, la población alicantina optó sobre todo por su propia cocina tradicional, pero no renunció a preparar recetas de otros territorios del Estado español. Las razones de esta preferencia son diversas. En el caso de Asturias se justifica por la tendencia a elaborar fabada en las semanas de más frío ${ }^{38}$. En el caso del País Vasco porque actúa como referente gastronómico para todo el Estado, incluso a escala mundial ${ }^{39}$. Al contrario que Asturias, su presencia en las cocinas alicantinas ha sido a través de multitud de platos casi siempre de pescado (marmitako, bacalao al pil-pil, kokotxas, merluza a la vasca, etc.). Por último, el caso de Castilla-La Mancha se justificaría por el protagonismo que tiene en la cocina tradicional alicantina a través de gazpachos, migas, gachamigas, sobre todo en las comarcas alicantinas que lindan con aquella región ${ }^{22,40}$. En relación con las recetas de otros territorios que aparecen en las respuestas de las personas encuestadas, si el confinamiento se hubiese producido en meses más cálidos, la importancia de platos procedentes de otros territorios como Andalucía y Cataluña, con sus gazpacho, salmorejo, escalivada, etc., quizás hubiese sido mayor.

Por último, en línea con lo apuntado en otros trabajos sobre los cambios actitudinales que ha provocado la COVID-19 o los nuevos estilos de vida que podrían desarrollarse ${ }^{41}$, muchas de las personas encuestadas en el presente estudio cambiaron durante el confinamiento la percepción que tenían sobre algunos de los elementos que configuran la cadena alimentaria. La idea de revalorizar la producción, el comercio y el consumo de alimentos de proximidad o la de 
incrementar el autoabastecimiento, sintetiza muchas de esas nuevas percepciones. Se trata de resultados que muestran el interés que pueden adquirir iniciativas encaminadas a profundizar en la sustentabilidad del modelo alimentario ${ }^{42}$. Son respuestas que correlacionan entre sí para formar un factor que genéricamente podría denominarse toma de conciencia sobre el sistema alimentario.

Entre las limitaciones del trabajo, cabe mencionar, quizá, no haber articulado todos los resortes necesarios para obtener una muestra representativa de personas encuestadas de otros territorios para poder comparar los resultados. También hay que señalar la condición de la herramienta utilizada como cuestionario ad hoc. Con más tiempo, se debería haber buscado su conexión con estudios precedentes para incluir indicadores directamente comparables.

Una de las fortalezas más destacada del estudio, ha sido la incorporación de las transformaciones producidas en las prácticas culinarias y gastronómicas en la herramienta de recogida de información del presente trabajo, ya que su análisis ha permitido mostrar la importancia de conocer las actividades culinarias y los hábitos gastronómicos para explicar los cambios en el comportamiento alimentario durante el confinamiento. Así mismo, muchos de los cambios relacionados con la mejora de las actitudes de las personas encuestadas frente al hecho alimentario, pueden orientar futuras acciones educativas que los consoliden y potencien. 


\section{CONCLUSIONES}

El confinamiento provocado por la COVID-19 ha dado lugar a cambios en las prácticas culinarias y gastronómicas de la población alicantina que pueden ayudar a alcanzar el objetivo de una alimentación más sabrosa, saludable y sostenible. El aumento de la actividad culinaria se tradujo en una apuesta por la cocina tradicional en detrimento de los platos precocinados, más convivialidad en el acto de comer y un mayor compromiso con el comercio y el consumo de alimentos de proximidad y la sustentabilidad del sistema alimentario. En futuras investigaciones habría que plantear la posibilidad de repetir el estudio tras el final de la pandemia, con el fin de conocer en qué medida persisten o se han modificado los cambios detectados. 


\section{CONTRIBUCIÓN DE AUTORÍA}

MTS, LCO participaron en la concepción y diseño de la metodología del trabajo, en la adquisición de datos y el análisis e interpretación de los mismos, en la redacción del texto y en la revisión final. APR participó en el diseño de la metodología del trabajo, en el análisis e interpretación de los datos, en la redacción del texto y en la revisión final. ARG participó en la concepción del trabajo, en la interpretación de los datos y en la revisión final. JBM participó en la concepción del trabajo, en el análisis e interpretación de los datos, en la redacción del texto y en la revisión final.

\section{FINANCIACIÓN}

Este trabajo fue financiado por la Cátedra Carmencita de Estudios del Sabor Gastronómico y el Centre de Gastronomía del Mediterrani UA-Dénia (Gasterra).

\section{CONFLICTO DE INTERESES}

Los autores expresan que no existen conflictos de interés al redactar el manuscrito. 


\section{REFERENCIAS}

(1) Organización Mundial de la Salud (OMS). Cronología de la respuesta de la OMS a la COVID-19 [Internet]. 2020 [citado 1 de octubre de 2020]. Disponible en: https://www.who.int/es/ news-room/detail/29-06-2020-covidtimeline

(2) Boletín Oficial del Estado (BOE). Ministerio de la Presidencia. Real Decreto 463/2020. España. 2020;67(I):25390-400. Disponible en: https://www.boe.es/boe/dias/2020/03/14/pdfs/ BOE-A-2020-3692.pdf

(3) Binder K, Diaz Crego M, Eckert G, Kotanidis S, Manko R, Monte M Del. States of emergency in response to the coronavirus crisis: Situation in certain Member States. 2020:1-12.

(4) Instituto Nacional de Estadística. Estimación del número de defunciones semanales durante el brote de COVID-19 [Internet]. 2020 [citado 25 de julio de 2020]. Disponible en: https://www.ine.es/experimental/defunciones/experimental_defunciones.htm\#tablas_resultado S

(5) Organización de Consumidores y Usuarios (OCU). Hábitos alimentarios durante el confinamiento. OCU [Internet]. 2020 Abr [citado el 15 de septiembre de 2020]. Disponible en: https://www.ocu.org/consumo-familia/derechos-consumidor/noticias/encuesta-habitoscoronavirus

(6) Díaz-Méndez C, García-Espejo I. Eating out in Spain: Motivations, sociability and consumer contexts. Appetite. 2017;119:14-22. DOI: https://doi.org/10.1016/j.appet.2017.03.047

(7) Asociación de Cadenas Españolas de Supermercados. Comunicado. trabajamos para dar el mejor servicio a la población en una situación excepcional [Internet]. Disponible en: http://www.asociacionsupermercados.com/resources/files/7b5c4490-39f5-40d3-86b1-

572a5f052d71.pdf

(8) Ministerio de Agricultura, Pesca y Alimentación. El Ministerio de Agricultura, Pesca y Alimentación constata una moderación en el volumen de compra de alimentos. 2020;1-4. Disponible en: https://www.mapa.gob.es/es/prensa/ultimas-noticias/el-ministerio-deagricultura-pesca-y-alimentaci\%C3\%B3n-constata-una-moderaci\%C3\%B3n-en-el-volumen-decompra-de-alimentos-/tcm:30-536857

(9) Pérez-Rodrigo C, Citores MG, Hervás Bárbara G, Litago FR, Casis Sáenz L, ArancetaBartrina J, et al. Cambios en los hábitos alimentarios durante el periodo de confinamiento por la pandemia COVID-19 en España. Rev Esp Nutr Comunitaria. 2020;26(2):28010. DOI: https://doi.org/10.14642/RENC.2020.26.2.5213 
(10) Rodríguez-Pérez C, Molina-Montes E, Verardo V, Artacho R, García-Villanova B, GuerraHernández EJ, et al. Changes in dietary behaviours during the COVID-19 outbreak confinement in the Spanish COVIDiet study. Nutrients. 2020;12(6):1-19. DOI: https://doi.org/10.3390/nu12061730

(11) Romeo-Arroyo E, Mora M, Vázquez-Araújo L. Consumer behavior in confinement times: Food choice and cooking attitudes in Spain. Int J Gastron Food Sci. 2020;21. DOI: https://doi.org/ 10.1016/j.ijgfs.2020.100226

(12) De Moraes Prata Gaspar MC, Ruiz Pascua M, Begueria A, Anadon S, Barba Martinez A, Larrea-Killinger C. Comer en tiempos de confinamiento: gestión de la alimentación, disciplina y placer. Perifèria, Rev Recer i Form en Antropol. 2020;25(2):63-73. DOI: https://doi.org/10.5565/rev/periferia.764

(13) Sidor A, Rzymski P. Dietary Choices and Habits during COVID-19 Lockdown: Experience from Poland. Nutrients. 2020;12(6):1657. DOI: https://doi.org/10.3390/nu12061657

(14) Di Renzo L, Gualtieri P, Pivari F, Soldati L, Attinà A, Cinelli G, et al. Eating habits and lifestyle changes during COVID-19 lockdown: An Italian survey. J TransI Med. 2020;18(1):229. DOI: https://doi.org/10.1186/s12967-020-02399-5

(15) Scarmozzino F, Visioli F. Covid-19 and the Subsequent Lockdown Modified Dietary Habits of Almost Half the Population in an Italian Sample. Foods. 2020;9(5):675. DOI: https://doi.org/10.3390/foods9050675

(16) Sudriá ME, Andreatta MM, Defagó MD. Los efectos de la cuarentena por coronavirus (COVID-19) en los hábitos alimentarios en Argentina. DIAETA. 2020;38(171):10-19.

(17) Varela Moreiras G, Serra Majem L, Aranceta Bartrina J. Nutrición y gastronomía en las distintas comunidades autónomas. La gastronomía segoviana en el contexto nacional. Conclusiones. Nutr Hosp. 2019;36(Extra 1):139-41. DOI: http://dx.doi.org/10.20960/nh.02729

(18) INE. Encuesta sobre equipamiento y uso de tecnologías de información y comunicación en hogares. Inst Nac Estadística [Internet]. 2019;2019:1-11. Disponible en: https://www.ine.es/prensa/tich_2019.pdf

(19) Knapp TR. Why Is the One-Group Pretest-Posttest Design Still Used? Clin Nurs Res. 2016; 25(5):467-72. DOI: https://doi.org/10.1177/1054773816666280

(20) Coll-Planas L, del Valle Gómez G, Bonilla P, Masat T, Puig T, Monteserin R. Promoting social capital to alleviate loneliness and improve health among older people in Spain. Heal Soc Care Community. 2017; 25(1):145-57. DOI: https://doi.org/10.1111/hsc.12284 
(21) Català-Oltra L. Problemas en la calidad de los alimentos y factores que facilitan la resistencia frente al patrón global alimentario. En: Penalva Verdú C, Ramos Feijoo C, editores. La construcción de la paz: propuestas multidisciplinares. Alicante: Universitat d'Alacant; 2008. p. 205-23.

(22) Bernabeu-mestre J, Quiles Izquierdo J, Galiana-Sánchez ME, Trescastro-López EM, Tormo. Nutrición y gastronomía en la Comunitat Valenciana. Nutr Hosp. 2019;36(1):86-91. DOI: http://dx.doi.org/10.20960/nh.02700

(23) Partearroyo T, Laja García Al, Varela-Moreiras G. Fortalezas y debilidades de la alimentación en la población española del siglo XXI. Nutr Hosp. 2019;4-7. DOI: http://dx.doi.org/ 10.20960/nh.02685

(24) Cal Fernández M, Garciá Mayor R V. Adherencia a la dieta Mediterránea en una muestra de la población adulta del sur de Galicia. Nutr Clin y Diet Hosp. 2017;37(3):95-7. DOI: 10.12873/373garcia

(25) Maiz E, Urdaneta E, Arillot X. La importancia de involucrar a niños y niñas en la preparación de las comidas. Nutr Hosp. 2018;35:136-9. DOI: http://dx.doi.org/10.20960/nh.2139

(26) García-González Á, Achón M, Alonso-Aperte E, Varela-Moreiras G. Identifying factors related to food agency: Cooking habits in the Spanish adult population-A cross-sectional study. Nutrients. 2018;10(2).DOI: https://doi.org/10.3390/nu10020217

(27) Taberner Guasp J. Sociología y educación: El sistema educativo en sociedades modernas. Funciones, cambios y conflictos. Tecnos, editor. Madrid; 2008.

(28) Bernabeu-Mestre J. La tapa como expresión de un estilo de convivencia: ¿una oportunidad para la comensalidad? Rev Española Cult Gastron. 2018;27-36. Disponible en: https://realacademiadegastronomia.com/revista/

(29) Laran J, Salerno A. Life-history strategy, food choice, and caloric consumption. Psychol Sci. 2013; 24(2):167-73. DOI: https://doi.org/10.1177/0956797612450033

(30) Balluerka Lasa, Nekane et al. Las consecuencias psicológicas de la COVID-19 y el confinamiento. Informe de investigación. Disponible en: https://www.ciencia.gob.es/stfls/MICINN/Universidades/Ficheros/Consecuencias_psicologicasC OVID19.pdf

(31) Fernández Ó. Internet y confinamiento: 79 horas de conexión a la semana, compras en horario laboral, películas y series. 20 minutos [Internet]. 2020; Disponible en: 
https://www.20minutos.es/noticia/4249527/0/internet-y-confinamiento-79-horas-de-conexiona-la-semana-compras-en-horario-laboral-peliculas-y-series/

(32) González-Soltero R, Blanco M], Biscaia JM, Mohedano RB, Grille-Mariscal M, Blanco MA. Análisis del contenido, posicionamiento y calidad de páginas web en español relacionadas con la nutrición y los trastornos de la conducta alimentaria. Nutr Hosp. 2015;31(3):1394-402. DOI: http://dx.doi.org/10.3305/nh.2015.31.3.8122

(33) Martín ISM, Fernández MG, Yurrita LC. Aplicaciones móviles en nutrición, dietética y hábitos saludables; análisis y consecuencia de una tendencia a la alza [Internet]. Vol. 30, Nutricion Hospitalaria. Sociedad Española de Nutrición Parenteral y Enteral (SENPE); 2014 [citado 30 de julio de 2020]. p. 15-24. DOI: http://dx.doi.org/10.3305/nh.2014.30.1.7398

(34) Varela Moreiras G. Gastronomía, comunicación y salud. Influencia de la gastronomía en los hábitos alimentarios de la población. Rev Española Comun En Salud. 2016;7(Sup1):85-94. DOI: http://dx.doi.org/10.20318/recs.2016.3125

(35) De Labarre M. Modernidad y alimentación: ¿hacia una «aculturación culinaria»? Quad la Mediterrània $=$ Cuad del Mediterráneo [Internet]. 2001;(2-3):189-98. Disponible en: https://dialnet.unirioja.es/servlet/articulo?codigo=2029884

(36) Richards G. Evolving Gastronomic Experiences: From Food to Foodies to Foodscapes. J Gastron Tour. 2015;1(1):5-17. DOI: https://doi.org/10.3727/216929715X14298190828796

(37) Getz D, Robinson RNS, Andersson TD, Vujicic S. Foodies and Food Tourism. Tourism Culture \& Communication. Goodfellow; 2014.

(38) Méndez Riestra E, Fernández-García B, Farias P, Galán N, Díaz-Méndez C, IglesiasGutiérrez E. Nutrición y gastronomía en el Principado de Asturias. Nutr Hosp 2019;36(N. Extra 1):14-20. DOI: http://dx.doi.org/10.20960/nh.02687

(39) Aranceta Bartrina J, Pérez-Rodrigo C. Nutrición y gastronomía en el País Vasco. Nutr Hosp 2019;36 (N.o Extra 1):29-38. DOI: http://dx.doi.org/10.20960/nh.02689

(40) Martínez García RM, García Perea A. Peculiaridades gastronómicas y modelo alimentario de la comunidad de Castilla-La Mancha. Nutr Hosp 2019;36(N. Extra 1):105-109. DOI: http://dx.doi.org/10.20960/nh.02705

(41) Tarhuni D, Hernandéz-Stefanoni J, Posada J, Nepote A, Varguez M. Huertos urbanos ... ¿fenómeno pasajero o nuevo estilo de vida ante la pandemia de la COVID-19? Cicy. 2020;12:11927. 
(42) Luyando JR. Conciencia social y ecològica en el consumoLuyando Cuevas,. Estud Soc [Internet]. 2016;25(47):303-23. Disponible en: http://www.redalyc.org/articulo.oa? id $=41744004012$ 